\title{
Effect of Integrated Nutrient Modules on Growth, Yield and Quality Parameters of Banana cv. Grand Naine
}

\author{
T. Ganapathi* and P.R. Dharmatti \\ Department of Horticulture, University of Agricultural Sciences, Dharwad-580 005, \\ Karnataka, India \\ *Corresponding author
}

\begin{abstract}
A B S T R A C T
Keywords

Banana, INM,

Nutrient module,

Soil properties and

NPK content in

plant

Article Info

Accepted:

14 December 2017

Available Online:

10 January 2018

A field experiment was conducted at new orchard, Department of Horticulture, University of Agricultural Sciences, Dharwad during 2014-15 and 2015-16 on "Integrated nutrient management studies in banana cv. Grand Naine (AAA)" through application of different combination of organic manures and inorganic fertilizers with green manure, Azospirillum and PSB. The results revealed that, Vermicompost equivalent to $40 \% \mathrm{RDN}(24.20 \mathrm{t} / \mathrm{ha})+$ Urea equivalent to $40 \% \operatorname{RDN}(535.73 \mathrm{~kg} / \mathrm{ha})+$ Green manure (sunnhemp @ $8.88 \mathrm{t} / \mathrm{ha})$ and Azospirillum (@30.86 kg/ha) equivalent to $20 \%$ RDN + PSB (@ $30.86 \mathrm{~kg} / \mathrm{ha}$ ) $\left(\mathrm{T}_{7}\right.$ ) recorded the highest growth parameters [pseudostem height $(205.05 \mathrm{~cm})$, pseudostem girth $(27.47 \mathrm{~cm})$, number of leaves $(16.00)$, leaf area $\left(8.87 \mathrm{~m}^{2}\right)$, leaf area index $(2.74)$, the least number of days taken for shooting (190.65) and total crop duration(318.89)], yield parameters [bunch weight $(26.94 \mathrm{~kg})$, number of hands per bunch (11.75), finger weight $(137.38 \mathrm{~g})$, finger length $(17.75 \mathrm{~cm})$, plot yield $(85.57 \mathrm{~kg})$, yield per ha $(66.02 \mathrm{t})]$ and quality parameters [TSS $\left(23.52^{0}\right.$ brix), total sugars $(20.30 \%)$, reducing sugars $(20.30 \%)$, non-reducing sugars (17.87\%), pulp to peel ratio (3.81) shelf life (6.33 days) and the least starch $(3.67 \%)$ and titrable acidity $(0.25)]$ followed by $\mathrm{T}_{10}$ (Agrigold combination) and $\mathrm{T}_{11}$ (Bhumilabh combination) treatments.
\end{abstract}

\section{Introduction}

In India, banana is cultivated in an area of 0.83 million ha with a production of 30 million tonnes (Anon., 2011).

The major banana growing areas are in Tamil Nadu, Maharashtra, Andhra Pradesh, Gujarat, Kerala, Karnataka, West Bengal and Orissa. It is being grown in an area of 1.12 lakh ha with a production of 2.28 lakh tonnes in Karnataka state.
Banana is known to consume more nutrients for its growth, yield and biomass production (Hazarika et al., 2015).

The use of chemical fertilizers alone has deleterious effect on soil physical, chemical and biological properties and productivity in the long run. The availability of organic manures is also one of the limitations for use in banana production. To fulfil the nutrient requirement only through organic or inorganic or bio- fertilizers alone is seldom possible but 
integration of all these sources will not only aid in achieving higher yields but also in sustaining the soil fertility status in the long run.

\section{Materials and Methods}

The field experiment with an integrated nutrient module consisting of organic manures, chemical fertilizers, green manure and bio-fertilizers in banana cv. Grand Naine was conducted during 2014-15 and 2015-16 at new orchard, Department of Horticulture, University of Agricultural Sciences, Dharwad. The soil type was red with clay texture.

The experiment consists of 12 treatments viz.

\section{Plant crop}

$\mathrm{T}_{1-}$ FYM equivalent to $40 \% \mathrm{RDN}$ (48.40 t/ha) + VC equivalent to $40 \% \mathrm{RDN}(24.20 \mathrm{t} / \mathrm{ha})+$ GM (Sunnhemp @ 8.88 t/ha) and Azospirillum $(30.86 \mathrm{~kg} / \mathrm{ha})$ equivalent to $20 \% \mathrm{RDN}+\mathrm{PSB}$ (30.86 kg/ha).

$\mathrm{T}_{2}$-FYM equivalent to $40 \% \mathrm{RDN}$ (48.40 t/ha) + PM equivalent to $40 \% \operatorname{RDN}(8.96 \mathrm{t} / \mathrm{ha})+$ GM (Sunnhemp @ 8.88 t/ha) and Azospirillum $(30.86 \mathrm{~kg} / \mathrm{ha})$ equivalent to $20 \% \mathrm{RDN}+\mathrm{PSB}$ (30.86 kg/ha).

$\mathrm{T}_{3-}$ FYM equivalent to $40 \% \mathrm{RDN}$ (48.40 t/ha) + SM equivalent to $40 \% \mathrm{RDN}(10.17 \mathrm{t} / \mathrm{ha})+$ GM (Sunnhemp @ 8.88 t/ha) and Azospirillum $(30.86 \mathrm{~kg} / \mathrm{ha})$ equivalent to $20 \% \mathrm{RDN}+\mathrm{PSB}$ (30.86 kg/ha).

$\mathrm{T}_{4-}$ FYM equivalent to $40 \% \mathrm{RDN}(48.40 \mathrm{t} / \mathrm{ha})$ + AG equivalent to $40 \%$ RDN (10.52 t/ha) + GM (Sunnhemp @ 8.88 t/ha) and Azospirillum $(30.86 \mathrm{~kg} / \mathrm{ha})$ equivalent to $20 \% \mathrm{RDN}+\mathrm{PSB}$ (30.86 kg/ha).

$\mathrm{T}_{5-}$ FYM equivalent to $40 \% \mathrm{RDN}(48.40 \mathrm{t} / \mathrm{ha})$ $+\mathrm{BL}$ equivalent to $40 \% \operatorname{RDN}(7.56 \mathrm{t} / \mathrm{ha})+$
GM (Sunnhemp @ 8.88 t/ha) and Azospirillum $(30.86 \mathrm{~kg} / \mathrm{ha})$ equivalent to $20 \% \mathrm{RDN}+\mathrm{PSB}$ (30.86 kg/ha).

$\mathrm{T}_{6-}$ FYM equivalent to $40 \% \mathrm{RDN}$ (48.40 t/ha) $+40 \%$ RDN through chemical fertilizer (Urea 535.73 kg/ ha) + GM (Sunnhemp @ 8.88 t/ha) and Azospirillum (30.86 kg/ha) equivalent to $20 \% \mathrm{RDN}+\mathrm{PSB}(30.86 \mathrm{~kg} / \mathrm{ha})$.

$\mathrm{T}_{7-} \mathrm{VC}$ equivalent to $40 \% \mathrm{RDN}(24.20 \mathrm{t} / \mathrm{ha})+$ $40 \%$ RDN through chemical fertilizer (Urea $535.73 \mathrm{~kg} / \mathrm{ha})+\mathrm{GM}$ (Sunnhemp @ $8.88 \mathrm{t} / \mathrm{ha})$ and Azospirillum (30.86 kg/ha) equivalent to $20 \% \mathrm{RDN}+\mathrm{PSB}(30.86 \mathrm{~kg} / \mathrm{ha})$.

$\mathrm{T}_{8-} \mathrm{PM}$ equivalent to $40 \% \mathrm{RDN}(8.96 \mathrm{t} / \mathrm{ha})+$ $40 \%$ RDN through chemical fertilizer (Urea $535.73 \mathrm{~kg} / \mathrm{ha})+$ GM (Sunnhemp @ $8.88 \mathrm{t} / \mathrm{ha})$ and Azospirillum (30.86 kg/ha) equivalent to $20 \% \mathrm{RDN}+\mathrm{PSB}(30.86 \mathrm{~kg} / \mathrm{ha})$.

$\mathrm{T}_{9-} \mathrm{SM}$ equivalent to $40 \% \mathrm{RDN}(10.17 \mathrm{t} / \mathrm{ha})+$ $40 \% \mathrm{RDN}$ through chemical fertilizer (Urea $535.73 \mathrm{~kg} / \mathrm{ha})+\mathrm{GM}$ (Sunnhemp @ $8.88 \mathrm{t} / \mathrm{ha})$ and Azospirillum (30.86 kg/ha) equivalent to $20 \% \mathrm{RDN}+\mathrm{PSB}(30.86 \mathrm{~kg} / \mathrm{ha})$.

$\mathrm{T}_{10-} \mathrm{AG}$ equivalent to $40 \% \mathrm{RDN}(10.52 \mathrm{t} / \mathrm{ha})$ + $40 \%$ RDN through chemical fertilizer (Urea $535.73 \mathrm{~kg} / \mathrm{ha})+$ GM (Sunnhemp @ 8.88 t/ha) and Azospirillum (30.86 kg/ha) equivalent to $20 \% \mathrm{RDN}+\mathrm{PSB}(30.86 \mathrm{~kg} / \mathrm{ha})$

$\mathrm{T}_{11 \text { - }} \mathrm{BL}$ equivalent to $40 \% \mathrm{RDN}(7.56 \mathrm{t} / \mathrm{ha})+$ $40 \%$ RDN through chemical fertilizer (Urea $535.73 \mathrm{~kg} / \mathrm{ha})+\mathrm{GM}$ (Sunnhemp @ $8.88 \mathrm{t} / \mathrm{ha})$ and Azospirillum (30.86 kg/ha) equivalent to $20 \% \mathrm{RDN}+\mathrm{PSB}(30.86 \mathrm{~kg} / \mathrm{ha})$

$\mathrm{T}_{12}$-Control (RDF 200: 100: $300 \mathrm{~g}$ NPK /plant or 617.20:308.60: $925.80 \mathrm{~kg} \mathrm{NPK/ha} \mathrm{+} \mathrm{Farm}$ yard manure@ 40 t/ha)

The recommended dose of phosphorous and potash $(100 \mathrm{~g} / \mathrm{plant}$ and $300 \mathrm{~g} / \mathrm{plant}$ 
respectively) was supplied through DAP and MOP.

\section{Ratoon crop}

$\mathrm{RDF}=100: 50: 100 \mathrm{NPK} \mathrm{g} / \mathrm{plant}$ or 308.60:154.20: 308.60 kg NPK/ha) +FYM @ $20 \mathrm{t} / \mathrm{ha}$

Note: 1) FYM- Farm Yard Manure, VCVermicompost, PM-Poultry Manure, SMSheep Manure, AG-Agrigold, BL-Bhumilabh, GM-Green manure, PSB-Phosphate Solubilizing bacteria. Azospirillum (ACD-15) and PSB (Pseudomonas striata) were used for the study.

The application of organic manures, chemical fertilizers with green manure and bio-fertilizer will compensate the majority of the plant nutrition thereby increase the productivity of banana cv. Grand Naine. The influence of integrated nutrient module was assessed on growth, yield and quality parameters of banana. The results of the field experiments conducted during the year 2014-15 and 201516 are pooled and discussed.

\section{Results and Discussion}

The results of the field experiments conducted during the year 2014-15 and 2015-16 are pooled and discussed under different headings.

\section{Effect of integrated nutrient modules on banana growth parameters (Table 1 and 2)}

The influence of different integrated nutrient modules on growth parameters which caused the yield variations are discussed here under.

The results of the pooled mean indicated that, at shooting the highest plant height (205.05 $\mathrm{cm})$ was recorded in $\mathrm{T}_{7}$ \{Vermicompost equivalent to $40 \% \mathrm{RDN}(24.20 \mathrm{t} / \mathrm{ha})+$ Chemical fertilizer (Urea $535.73 \mathrm{~kg} / \mathrm{ha}$ ) equivalent to $40 \% \mathrm{RDN}+$ Green manure (sunnhemp @8.88 tons/ha) and Azospirillum (30.86 kg/ha) equivalent to $20 \% \mathrm{RDN}+\mathrm{PSB}$ $(30.86 \mathrm{~kg} / \mathrm{ha})\}$ which was on par with $\mathrm{T}_{6}, \mathrm{~T}_{10}$ and $\mathrm{T}_{11}$ and significantly superior over rest of the treatments. The least pseudostem height was recorded in $\mathrm{T}_{8}(171.53 \mathrm{~cm})$ followed by $\mathrm{T}_{3}$. The pseudostem girth was significantly higher with $\mathrm{T}_{7}$ treatment $(27.47 \mathrm{~cm})$ followed by $\mathrm{T}_{9}$ which was on par with $\mathrm{T}_{1}, \mathrm{~T}_{4}, \mathrm{~T}_{5}, \mathrm{~T}_{6}, \mathrm{~T}_{9}$, $\mathrm{T}_{10}, \mathrm{~T}_{11}$ and $\mathrm{T}_{12}$ and the least pseudostem girth $(24.99 \mathrm{~cm})$ was recorded in plants treated with $\mathrm{T}_{8}$. The highest number of leaves (16.00) was recorded in $\mathrm{T}_{7}$ \{Vermicompost equivalent to $40 \%$ of RDN (24.20 t/ha) + Chemical fertilizer (Urea $535.73 \mathrm{~kg} / \mathrm{ha}$ ) equivalent to 40 $\%$ RDN + Green manure (sunnhemp @ 8.88 tons/ha) and Azospirillum (30.86 $\mathrm{kg} / \mathrm{ha})$ equivalent to $20 \% \mathrm{RDN}+\mathrm{PSB}$ (30.86 $\mathrm{kg} / \mathrm{ha}$ ) $\}$ indicating the superiority of integrated nutrient module over organic treatments alone which was significantly superior over all other treatments except $\mathrm{T}_{3}$ and $\mathrm{T}_{8}$. The least number of leaves (13.24) was recorded in $\mathrm{T}_{8}$ (Table 1).

The application vermicompost equivalent to $40 \% \mathrm{RDN}$ (24.20 t/ha) + Chemical fertilizer (Urea $535.73 \mathrm{~kg} / \mathrm{ha}$ ) equivalent to $40 \% \mathrm{RDN}$ + Green manure (sunnhemp @ 8.88 tons/ha) + Azospirillum $(30.86 \mathrm{~kg} / \mathrm{ha})$ equivalent to $20 \%$ $\mathrm{RDN}+\mathrm{PSB}(30.86 \mathrm{~kg} / \mathrm{ha})\left(\mathrm{T}_{7}\right)$ recorded significantly higher leaf area $\left(8.87 \mathrm{~m}^{2}\right)$ followed by $\mathrm{T}_{1}, \mathrm{~T}_{4}, \mathrm{~T}_{5}, \mathrm{~T}_{9}, \mathrm{~T}_{10}$ and $\mathrm{T}_{11}$. The integrated nutrient modules recorded the higher leaf area as compared to organic nutrient modules except in $\mathrm{T}_{8}$. The pooled data indicated that, the highest leaf area index (2.74) at shooting was recorded in $\mathrm{T}_{7}$ which was on par with $T_{1}, T_{4}, T_{5}, T_{9}, T_{10}$ and $T_{11}$ treatments having integrated nutrient modules and superior over rest of the treatments. The mean least number of days taken for shooting, shooting to harvest and total crop duration (190.65, 128.24 and 318.89 days respectively) was recorded in $\mathrm{T}_{7}$ \{ Vermicompost equivalent to $40 \% \operatorname{RDN}(24.20 \mathrm{t} / \mathrm{ha})+$ Chemical 
fertilizer (Urea $535.73 \mathrm{~kg} / \mathrm{ha}$ ) equivalent to 40 $\%$ RDN + Green manure (sunnhemp @ 8.87 t/ha) and Azospirillum (30.86 kg/ha) equivalent to $20 \% \mathrm{RDN}+\mathrm{PSB}(30.86 \mathrm{~kg} / \mathrm{ha})$ (Table 2).

The results indicated that, the integrated nutrient modules $\mathrm{T}_{7}$ (Vermicompost combination), $\mathrm{T}_{10}$ (Agrigold combination) and $\mathrm{T}_{11}$ (Bhumilabh combination) were recorded lesser days taken for shooting, shooting harvest and total crop duration indicating the potentiality of these organic manures along with chemical and bio-fertilizers. These integrated treatments were found to be most promising in obtaining higher growth parameters of banana.

The similar results were also obtained by several other workers. Jayabaskaran et al., (2001) observed significantly higher plant height in Poovan banana by application of poultry manure at $15 \mathrm{~kg}$ per plant and followed by application of rice husk ash @ 15 $\mathrm{kg}$ per plant. Nachegowda et al., (2004) recorded that the plants applied with 180:108:220 $\mathrm{g}$ of NPK $+15 \mathrm{~kg}$ of FYM were recorded the highest plant height followed by banana plants supplied with $2.5 \mathrm{~kg}$ sheep manure +180:108:220 g NPK/plant/year.

Patil and Shinde (2013) also reported that, treatment with $50 \%$ RDF + FYM (10 $\mathrm{kg} /$ plant $)+$ Azotobacter (50 g/Plant) + PSB (50 g/plant) + VAM (250 g/plant) recorded significantly the highest number of leaves at different days after planting and at shooting stage.

\section{Effect of integrated nutrient modules on banana yield parameters (Table 3 )}

The influence of different integrated nutrient modules on yield parameters which caused the yield variations are discussed here under.

The pooled data indicated that, the maximum mean yield of banana per hectare (66.02 t/ha) was recorded in $\mathrm{T}_{7}$ \{Vermicompost equivalent to $40 \% \operatorname{RDN}(24.20 \mathrm{t} / \mathrm{ha})+$ Chemical fertilizer (Urea $535.73 \mathrm{~kg} / \mathrm{ha}$ ) equivalent to 40 $\%$ RDN + Green manure (Sunnhemp @ 8.88 t/ha) + Azospirillum (30.86 kg/ha) equivalent to $20 \% \mathrm{RDN}+\mathrm{PSB}(30.86 \mathrm{~kg} / \mathrm{ha})\}$ which was on par with $\mathrm{T}_{1}, \mathrm{~T}_{10}$ and $\mathrm{T}_{11}$ and significantly superior over rest of the treatments (Table 3 ).

The highest mean yield of banana in $\mathrm{T}_{7}$ was attributed to the higher growth and yield contributing parameters in the same treatment. The mean yield components of banana indicated that the maximum bunch weight $(26.94 \mathrm{~kg})$, number of hands per bunch (11.75), finger weight $(137.38 \mathrm{~g})$, finger length $(17.75 \mathrm{~cm})$, number of fingers on third hand (16.93) were recorded in plants applied with vermicompost equivalent to $40 \% \mathrm{RDN}$ $(24.20 \mathrm{t} / \mathrm{ha})+$ Chemical fertilizer (Urea 535.73 $\mathrm{kg} / \mathrm{ha})$ equivalent to $40 \% \mathrm{RDN}+$ Green manure (sunnhemp @ 8.88 t/ha) and Azospirillum $(30.86 \mathrm{~kg} / \mathrm{ha})$ equivalent to $20 \%$ RDN + PSB (30.86 kg/ha) $\left(\mathrm{T}_{7}\right)$. The similar results were also recorded with integrated nutrient modules by many scientists in banana. Athani et al., (1999) reported that, maximum number of fingers per bunch, bunch weight $(5.10 \mathrm{~kg} / \mathrm{bunch})$ and finally fruit yield $(15.14$ t/ha) was obtained by applying $50 \% \mathrm{RDF}+$ vermicompost $2.00 \mathrm{~kg} / \mathrm{plant}$ in Rajapuri banana. They also reported that, organic matter in the form of vermicompost in combination with inorganic fertilizers increased the yield in banana cv. Rajapuri. Nachegowda et al., (2004) reported that, plants applied with $15 \mathrm{~kg}$ FYM + 180:108:220g NPK/plant/year recorded the highest bunch weight $(49.47 \mathrm{~kg})$, fruit length $(25.19 \mathrm{~cm})$, fruit weight $(220.21 \mathrm{~g})$, finger /hand (19.00), fingers/bunch (227.94) and the yield (148.41 t/ha) in banana followed by 2.5 $\mathrm{kg}$ Sheep manure + 108:108:2205g NPK /plant/year. 
Table.1 Influence of different sources of nutrient modules on growth parameters in banana cv. Grand Naine (Pooled mean)

\begin{tabular}{|c|c|c|c|c|c|c|c|c|c|c|c|c|c|c|c|}
\hline \multirow{2}{*}{$\begin{array}{l}\text { Treatm } \\
\text { ents }\end{array}$} & \multicolumn{5}{|c|}{ Pseudostem height (cm) } & \multicolumn{5}{|c|}{ Pseudostem girth (cm) } & \multicolumn{5}{|c|}{ Number of leaves } \\
\hline & $\begin{array}{l}35 \\
\text { DAP }\end{array}$ & $\begin{array}{l}70 \\
\text { DAP }\end{array}$ & $\begin{array}{l}105 \\
\text { DAP }\end{array}$ & $\begin{array}{l}140 \\
\text { DAP }\end{array}$ & $\begin{array}{l}\text { At } \\
\text { shooting }\end{array}$ & $\begin{array}{l}35 \\
\text { DAP }\end{array}$ & $\begin{array}{l}70 \\
\text { DAP }\end{array}$ & $\begin{array}{l}105 \\
\text { DAP }\end{array}$ & $\begin{array}{l}140 \\
\text { DAP }\end{array}$ & $\begin{array}{l}\text { At } \\
\text { shooting }\end{array}$ & $\begin{array}{l}35 \\
\text { DAP }\end{array}$ & $\begin{array}{l}70 \\
\text { DAP }\end{array}$ & $\begin{array}{l}105 \\
\text { DAP }\end{array}$ & $\begin{array}{l}140 \\
\text { DAP }\end{array}$ & $\begin{array}{l}\text { At } \\
\text { shooting }\end{array}$ \\
\hline $\mathrm{T}_{1}$ & 40.13 & 69.63 & 106.09 & 139.27 & 189.33 & 7.75 & 10.57 & 13.40 & 17.52 & 27.12 & 7.16 & 11.44 & 11.78 & 11.79 & 15.35 \\
\hline $\mathbf{T}_{2}$ & 35.08 & 66.93 & 102.84 & 136.71 & 183.31 & 6.46 & 10.12 & 13.04 & 16.86 & 25.25 & 6.70 & 11.37 & 11.81 & 11.32 & 15.03 \\
\hline $\mathbf{T}_{3}$ & 32.78 & 66.64 & 101.61 & 136.20 & 181.39 & 6.75 & 9.68 & 12.93 & 16.77 & 25.62 & 6.50 & 10.76 & 11.29 & 10.84 & 14.36 \\
\hline $\mathrm{T}_{4}$ & 38.47 & 69.83 & 104.38 & 137.78 & 186.27 & 7.33 & 10.35 & 13.31 & 17.42 & 26.62 & 6.01 & 11.26 & 11.71 & 11.46 & 15.18 \\
\hline $\mathrm{T}_{5}$ & 38.27 & 67.86 & 104.77 & 137.36 & 185.62 & 7.23 & 10.39 & 13.37 & 17.07 & 26.01 & 7.02 & 11.44 & 11.71 & 11.46 & 15.27 \\
\hline$T_{6}$ & 41.05 & 72.03 & 109.24 & 140.95 & 194.26 & 8.15 & 11.28 & 14.11 & 17.79 & 27.25 & 8.25 & 11.86 & 11.83 & 11.83 & 15.68 \\
\hline $\mathbf{T}_{7}$ & 51.96 & 83.65 & 117.54 & 146.97 & 205.05 & 9.26 & 11.97 & 14.85 & 18.16 & 27.47 & 9.37 & 12.42 & 12.33 & 11.90 & 16.00 \\
\hline $\mathrm{T}_{8}$ & 30.32 & 65.45 & 99.77 & 128.15 & 171.53 & 6.65 & 9.56 & 12.72 & 16.14 & 24.99 & 5.64 & 10.01 & 10.41 & 10.00 & 13.24 \\
\hline $\mathrm{T}_{9}$ & 40.33 & 71.32 & 106.32 & 139.02 & 190.44 & 7.95 & 11.03 & 13.43 & 17.60 & 27.26 & 8.06 & 11.64 & 11.76 & 11.39 & 15.53 \\
\hline$T_{10}$ & 46.75 & 79.32 & 116.31 & 146.49 & 203.25 & 8.76 & 11.83 & 14.81 & 17.81 & 26.85 & 8.62 & 12.25 & 12.27 & 11.87 & 15.80 \\
\hline$T_{11}$ & 42.53 & 75.04 & 109.36 & 142.29 & 196.96 & 8.62 & 11.60 & 14.30 & 17.80 & 26.77 & 8.35 & 11.96 & 12.07 & 11.61 & 15.45 \\
\hline $\mathrm{T}_{12}$ & 40.94 & 71.52 & 106.55 & 139.57 & 191.92 & 7.71 & 10.67 & 13.57 & 17.79 & 26.75 & 8.09 & 11.71 & 11.75 & 11.69 & 15.26 \\
\hline $\mathrm{S} \mathrm{Em+/-}$ & 4.75 & 4.72 & 3.42 & 3.57 & 4.16 & 0.71 & 0.47 & 0.60 & 0.51 & 0.43 & 0.22 & 0.38 & 0.34 & 0.36 & 0.51 \\
\hline $\begin{array}{c}\text { CD at } \\
5 \%\end{array}$ & 13.93 & 13.85 & 10.04 & 10.48 & 12.20 & 2.08 & 1.39 & 1.76 & 1.49 & 1.27 & 0.65 & 1.12 & 1.01 & 1.05 & 1.51 \\
\hline
\end{tabular}

DAP: Days after planting

T. FYM equivalent to $40 \%$ RDN (48.40 t/ha) + VC equivalent to $40 \%$ RDN (24.20 t/ha) + GM (sunnhemp @ 8.88 t/ha) and Azospirillum (@ $30.86 \mathrm{~kg} / \mathrm{ha}$ ) equivalent to $20 \%$ RDN + PSB (@ $30.86 \mathrm{~kg} / \mathrm{ha}$ ). T2-FYM equivalent to 40\% RDN (48.40 t/ha) + PM equivalent to 40\% RDN (8.96 t/ha) + GM (sunnhemp @ 8.88 t/ha) and Azospirillum (@30.86 kg/ha) equivalent to 20\% RDN + PSB (@ $30.86 \mathrm{~kg} / \mathrm{ha}$ ). 3. FYM equivalent to 40\% RDN (48.40 / ha) + SM equiant

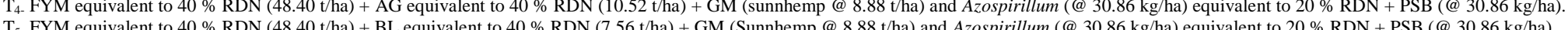
T 5 - FYM equivalent to $40 \%$ RDN (48.40 t/ha) + BL equivalent to $40 \%$ RDN (7.56 t/ha) + GM (Sunnhemp @ 8.88 t/ha) and Azospirillum (@ 30.86 kg/ha) equivalent to $20 \%$ RDN + PSB (@ $30.86 \mathrm{~kg} / \mathrm{ha}$ ). $\mathrm{T}_{6}$ FYM equivalent to $40 \%$ RDN (48.40 tha) + 40\% RDN through chemical fertilizer (urea $535.73 \mathrm{~kg} / \mathrm{ha}$ ) + GM (sunnhemp @ 8.88 tha) and Azospirillum (@30.86 kg/ha) equivalent to $20 \%$ RDN + PSB (@ $\left.30.86 \mathrm{~kg} / \mathrm{ha}\right)$. 7. VC equivalent to $40 \%$ RDN (24.20 /ha) + 40\% RDN through chemical fertilizer (urea $535.73 \mathrm{~kg} / \mathrm{ha}$ ) + GM (sunnhemp@ @ $8.88 \mathrm{tha}$ ) and Azospirillum (@ $30.86 \mathrm{~kg} / \mathrm{ha}$ ) equivalent to $20 \% \mathrm{RDN}+\mathrm{PSB}$ (@ $30.86 \mathrm{~kg} / \mathrm{ha}$ ). T8. PM equivalent to $40 \%$ RDN (8.96 t/ha)+ $40 \%$ RDN through chemical fertilizer (urea $535.73 \mathrm{~kg} / \mathrm{ha}$ ) + GM (sunnhemp @ 8.88 t/ha) and Azospirillum (@ $30.86 \mathrm{~kg} / \mathrm{ha}$ ) equivalent to $20 \%$ RDN + PSB (@30.86 kg/ha). T. SM equivalent to 40 \% RDN (10.17 t/ha) + 40\% RDN through chemical fertilizer (urea 535.73 kg/ha) + GM (sunnhemp @ 8.88 t/ha) and Azospirillum (@ $30.86 \mathrm{~kg} / \mathrm{ha}$ ) equivalent to 20\% RDN + PSB (@30.86 kg/ha). T10. AG equivalento 40 RDN (7.56 t/ha) + 40\% RDN 11- BLequin $T_{12}$-Control (RDF=617.20:308.60: $925.80 \mathrm{~kg} \mathrm{NPK} / \mathrm{ha}+$ Farm yard manure@ 40 t/ha (plant crop)

Ratoon crop: RDF=308.60:154.20: 308.60 kg NPK/ha) + FYM @ 20/ha accordingly it has been supplied through different sources

FYM- Farmyard manure, VC-Vermicompost, PM-Poultry manure, SM-Sheep manure, AG-Agrigold, BL-Bhumilabh, GM-Green manure, PSB-Phosphate Solubilizing bacteria 
Table.2 Influence of different sources of nutrient modules on growth parameters in banana cv. Grand Naine (Pooled mean)

\begin{tabular}{|c|c|c|c|c|c|c|c|c|c|c|c|c|c|}
\hline \multirow[t]{2}{*}{ Treatments } & \multicolumn{5}{|c|}{ Leaf area $\left(\mathrm{m}^{2}\right)$} & \multicolumn{5}{|c|}{ Leaf area Index } & \multicolumn{3}{|c|}{ Days } \\
\hline & $\begin{array}{l}35 \\
\text { DAP }\end{array}$ & $\begin{array}{l}70 \\
\text { DAP }\end{array}$ & $\begin{array}{l}105 \\
\text { DAP }\end{array}$ & $\begin{array}{l}140 \\
\text { DAP }\end{array}$ & $\begin{array}{l}\text { At } \\
\text { shooting }\end{array}$ & $\begin{array}{l}35 \\
\text { DAP }\end{array}$ & $\begin{array}{l}70 \\
\text { DAP }\end{array}$ & $\begin{array}{l}105 \\
\text { DAP }\end{array}$ & $\begin{array}{l}140 \\
\text { DAP }\end{array}$ & $\begin{array}{l}\text { At } \\
\text { shooting }\end{array}$ & $\begin{array}{l}\text { Taken for } \\
\text { shooting }\end{array}$ & $\begin{array}{l}\text { Shooting } \\
\text { to } \\
\text { harvest }\end{array}$ & $\begin{array}{l}\text { Total } \\
\text { crop } \\
\text { duration }\end{array}$ \\
\hline$T_{1}$ & 1.09 & 2.35 & 3.01 & 3.32 & 7.52 & 0.34 & 0.72 & 0.93 & 1.25 & 2.32 & 215.24 & 133.88 & 339.12 \\
\hline $\mathbf{T}_{2}$ & 0.96 & 2.31 & 2.39 & 3.17 & 6.30 & 0.30 & 0.71 & 0.74 & 1.02 & 1.94 & 226.91 & 138.08 & 364.99 \\
\hline $\mathbf{T}_{3}$ & 0.90 & 2.07 & 2.38 & 3.83 & 6.03 & 0.28 & 0.64 & 0.73 & 0.98 & 1.86 & 229.15 & 138.41 & 367.56 \\
\hline $\mathrm{T}_{4}$ & 0.77 & 2.27 & 2.85 & 3.72 & 7.25 & 0.24 & 0.70 & 0.88 & 1.18 & 2.24 & 220.96 & 134.95 & 355.91 \\
\hline $\mathbf{T}_{5}$ & 1.05 & 2.33 & 2.85 & 3.53 & 7.09 & 0.32 & 0.72 & 0.88 & 1.15 & 2.19 & 226.42 & 136.89 & 363.30 \\
\hline$T_{6}$ & 1.45 & 2.52 & 3.12 & 4.58 & 6.69 & 0.45 & 0.78 & 0.96 & 1.09 & 2.07 & 204.46 & 135.50 & 339.96 \\
\hline$T_{7}$ & 1.89 & 2.82 & 3.65 & 2.81 & 8.87 & 0.58 & 0.87 & 1.13 & 1.41 & 2.74 & 190.65 & 128.24 & 318.89 \\
\hline $\mathbf{T}_{8}$ & 0.68 & 1.83 & 2.13 & 3.87 & 5.30 & 0.21 & 0.57 & 0.66 & 0.87 & 1.63 & 230.66 & 138.50 & 369.16 \\
\hline $\mathbf{T}_{9}$ & 1.39 & 2.41 & 3.01 & 4.44 & 7.66 & 0.43 & 0.74 & 0.93 & 1.19 & 2.36 & 211.41 & 132.39 & 343.80 \\
\hline$T_{10}$ & 1.58 & 2.75 & 3.50 & 4.41 & 8.57 & 0.49 & 0.85 & 1.08 & 1.37 & 2.64 & 195.25 & 128.26 & 323.51 \\
\hline$T_{11}$ & 1.49 & 2.55 & 3.39 & 3.66 & 8.39 & 0.46 & 0.79 & 1.05 & 1.36 & 2.59 & 201.09 & 129.16 & 330.24 \\
\hline $\mathrm{T}_{12}$ & 1.40 & 2.44 & 3.15 & 3.32 & 6.83 & 0.43 & 0.75 & 0.97 & 1.13 & 2.11 & 206.93 & 131.67 & 338.59 \\
\hline S Em+/- & 0.08 & 0.16 & 0.29 & 0.33 & 0.63 & 0.02 & 0.05 & 0.09 & 0.10 & 0.20 & 0.80 & 0.55 & 1.21 \\
\hline CD at $5 \%$ & 0.24 & 0.46 & 0.85 & 0.96 & 1.86 & 0.07 & 0.14 & 0.26 & 0.30 & 0.57 & 2.36 & 1.62 & 3.55 \\
\hline
\end{tabular}

DAP: Days after planting

T. FYM equivalent to 40 \% RDN (48.40 t/ha) + VC equivalent to 40 \% RDN (24.20 t/ha) + GM (sunnhemp @ 8.88 t/ha) and Azospirillum (@ $30.86 \mathrm{~kg} / \mathrm{ha}$ ) equivalent to 20\% RDN + PSB (@ 30.86 kg/ha). T2-FYM equivalent to $40 \%$ RDN (48.40 t/ha) + PM equivalent to 40 \% RDN (8.96 t/ha) + GM (sunnhemp @ 8.88 t/ha) and Azospirillum (@ $30.86 \mathrm{~kg} / \mathrm{ha}$ ) equivalent to $20 \%$ RDN + PSB (@ $30.86 \mathrm{~kg} / \mathrm{ha}$ ). $\mathrm{T}_{3}$ - FYM equivalent to $40 \%$ RDN (48.40 t/ha) + SM equivalent to 40 \% RDN (10.17 t/ha) + GM (sunnhemp @ 8.88 t/ha) and Azospirillum (@ $30.86 \mathrm{~kg} / \mathrm{ha}$ ) equivalent to $20 \%$ RDN + PSB (@ $30.86 \mathrm{~kg} / \mathrm{ha}$ ). $\mathrm{T}_{4}$ - FYM equivalent to $40 \% \mathrm{RDN}$ (48.40 t/ha) + AG equivalent to 40\% RDN (10.52 t/ha) + GM (sunnhemp @ 8.88 t/ha) and Azospirillum (@30.86 kg/ha) equivalent to $20 \%$ RDN + PSB (@30.86 kg/ha).

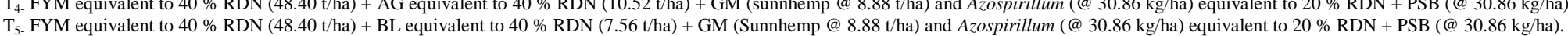
$\mathrm{T}_{6}$ FYM equivalent to $40 \%$ RDN (48.40 t/ha) + $40 \%$ RDN through chemical fertilizer (urea $535.73 \mathrm{~kg} / \mathrm{ha}$ ) + GM (sunnhemp @ 8.88 t/ha) and Azospirillum (@ $30.86 \mathrm{~kg} / \mathrm{ha}$ ) equivalent to $20 \%$ RDN + PSB (@30.86 kg/ha). T 7. VC equivalent to $40 \%$ RDN (24.20 t/ha) + 40 \% RDN through chemical fertilizer (urea $535.73 \mathrm{~kg} / \mathrm{ha}$ ) + GM (sunnhemp@ 8.88 t/ha) and Azospirillum (@ $30.86 \mathrm{~kg} / \mathrm{ha}$ ) equivalent to $20 \%$ RDN + PSB (@ 30.86 kg/ha). T. PM equivalent to $40 \%$ RDN (8.96 t/ha)+ $40 \%$ RDN through chemical fertilizer (urea $535.73 \mathrm{~kg} / \mathrm{ha}$ ) + GM (sunnhemp @8.88 t/ha) and Azospirillum (@ $30.86 \mathrm{~kg} / \mathrm{ha}$ ) equivalent to $20 \%$ RDN + PSB (@ $30.86 \mathrm{~kg} / \mathrm{ha}$ ). T. SM equivalent to $40 \%$ RDN (10.17 t/ha) + 40 \% RDN through chemical fertilizer (urea $535.73 \mathrm{~kg} / \mathrm{ha}$ ) + GM (sunnhemp @ 8.88 t/ha) and Azospirillum (@30.86 kg/ha) equivalent to $20 \%$ RDN + PSB (@30.86 kg/ha). $\mathrm{T}_{10}$ - AG equivalent to $40 \% \mathrm{RDN}$ (10.52 t/ha) + 40\% RDN through chemical fertilizer (urea $535.73 \mathrm{~kg} / \mathrm{ha}$ ) + GM (sunnhemp @8 8.88 t/ha) and Azospirillum (@30.86 kg/ha) equivalent to $20 \%$ RDN + PSB (@30.86 kg/ha $\mathrm{T}_{11}$ - BL equivalent to $40 \%$ RDN (7.56 t/ha) + 40\% RDN through chemical fertilizer (urea $535.73 \mathrm{~kg} / \mathrm{ha}$ ) + GM (sunnhemp @ 8.88 t/ha) and Azospirillum (@ $30.86 \mathrm{~kg} / \mathrm{ha}$ ) equivalent to $20 \%$ RDN + PSB (@30.86 kg/ha) $\mathrm{T}_{12}$-Control (RDF=617.20:308.60: $925.80 \mathrm{~kg} \mathrm{NPK/ha}+$ Farm yard manure@ $40 \mathrm{t} / \mathrm{ha}$ (plant crop)

Ratoon crop: RDF=308.60:154.20: $308.60 \mathrm{~kg} \mathrm{NPK/ha)} \mathrm{+} \mathrm{FYM} \mathrm{@} \mathrm{20/ha} \mathrm{accordingly} \mathrm{it} \mathrm{has} \mathrm{been} \mathrm{supplied} \mathrm{through} \mathrm{different} \mathrm{sources}$

FYM- Farmyard manure, VC-Vermicompost, PM-Poultry manure, SM-Sheep manure, AG-Agrigold, BL-Bhumilabh, GM-Green manure, PSB-Phosphate Solubilizing bacteria 
Table.3 Influence of different sources of nutrient modules on yield parameters in banana cv. Grand Naine (Pooled mean)

\begin{tabular}{|c|c|c|c|c|c|c|c|}
\hline Treatments & $\begin{array}{l}\text { Bunch weight. } \\
\text { (kg) }\end{array}$ & $\begin{array}{l}\text { No. of hands } \\
\text { per bunch }\end{array}$ & $\begin{array}{l}\text { Finger weight. } \\
\text { (g) }\end{array}$ & $\begin{array}{l}\text { Finger length } \\
\text { (cm) }\end{array}$ & $\begin{array}{l}\text { No. of Fingers on } \\
3^{\text {rd }} \text { hand }\end{array}$ & Plot yield (kg) & Yield (t/ha) \\
\hline $\mathbf{T}_{1}$ & 18.17 & 10.06 & 124.27 & 16.14 & 14.89 & 73.21 & 56.48 \\
\hline $\mathbf{T}_{2}$ & 14.58 & 9.10 & 117.77 & 15.23 & 13.88 & 60.44 & 46.63 \\
\hline $\mathbf{T}_{3}$ & 11.55 & 8.58 & 104.28 & 14.36 & 13.10 & 58.35 & 45.02 \\
\hline $\mathbf{T}_{4}$ & 17.85 & 10.31 & 123.43 & 15.32 & 14.26 & 72.72 & 56.10 \\
\hline $\mathbf{T}_{5}$ & 16.41 & 9.73 & 120.39 & 15.55 & 14.19 & 70.04 & 54.04 \\
\hline$T_{6}$ & 19.18 & 10.02 & 129.53 & 16.12 & 15.02 & 70.52 & 54.40 \\
\hline $\mathbf{T}_{7}$ & 26.94 & 11.75 & 137.38 & 17.75 & 16.93 & 85.57 & 66.02 \\
\hline $\mathbf{T}_{8}$ & 12.85 & 9.54 & 104.43 & 14.40 & 13.09 & 61.69 & 47.59 \\
\hline $\mathbf{T}_{9}$ & 19.54 & 10.19 & 129.83 & 16.12 & 15.04 & 70.83 & 54.64 \\
\hline$T_{10}$ & 23.69 & 10.98 & 135.91 & 17.18 & 16.12 & 84.27 & 65.01 \\
\hline $\mathrm{T}_{11}$ & 22.51 & 10.49 & 135.16 & 17.07 & 16.14 & 73.66 & 56.83 \\
\hline $\mathrm{T}_{12}$ & 19.17 & 9.70 & 128.02 & 16.66 & 15.68 & 71.21 & 54.94 \\
\hline S Em+/- & 0.65 & 0.15 & 1.84 & 0.31 & 0.30 & 4.28 & 3.30 \\
\hline CD at $5 \%$ & 1.91 & 0.44 & 5.39 & 0.90 & 0.87 & 12.55 & 9.68 \\
\hline
\end{tabular}

T. FYM equivalent to 40 \% RDN (48.40 t/ha) + VC equivalent to 40 \% RDN (24.20 t/ha)+ GM (sunnhemp @ 8.88 t/ha) and Azospirillum (@ 30.86 kg/ha) equivalent to $20 \%$ RDN + PSB (@ 30.86 $\mathrm{kg} / \mathrm{ha})$.

T2-FYM equivalent to 40 \% RDN (48.40 t/ha) + PM equivalent to 40 \% RDN (8.96 t/ha) + GM (sunnhemp @ 8.88 t/ha) and Azospirillum (@ 30.86 kg/ha) equivalent to 20\% RDN + PSB (@ 30.86 $\mathrm{kg} / \mathrm{ha})$.

$\mathrm{T}_{3-}$ FYM equivalent to 40 \% RDN (48.40 t/ha) + SM equivalent to 40 \% RDN (10.17 t/ha) + GM (sunnhemp @ 8.88 t/ha) and Azospirillum (@ 30.86 kg/ha) equivalent to $20 \%$ RDN + PSB (@ 30.86 $\mathrm{kg} / \mathrm{ha}$ ).

T.-FYM equivalent to 40 \% RDN (48.40 t/ha) + AG equivalent to 40 \% RDN (10.52 t/ha) + GM (sunnhemp @ 8.88 t/ha) and Azospirillum (@ 30.86 kg/ha) equivalent to $20 \%$ RDN + PSB (@ 30.86 $\mathrm{kg} / \mathrm{ha})$.

T5. FYM equivalent to 40 \% RDN (48.40 t/ha) + BL equivalent to 40 \% RDN (7.56 t/ha)+GM (Sunnhemp @ 8.88 t/ha) and Azospirillum (@ 30.86 kg/ha) equivalent to $20 \%$ RDN + PSB (@ 30.86 $\mathrm{kg} / \mathrm{ha})$.

$\mathrm{T}_{6}$ FYM equivalent to $40 \%$ RDN (48.40 t/ha) + 40 \% RDN through chemical fertilizer (urea $535.73 \mathrm{~kg} / \mathrm{ha}$ ) + GM (sunnhemp @ 8.88 t/ha) and Azospirillum (@ $30.86 \mathrm{~kg} / \mathrm{ha}$ ) equivalent to $20 \%$ RDN + PSB (@ $30.86 \mathrm{~kg} / \mathrm{ha})$.

T7. VC equivalent to $40 \%$ RDN (24.20 t/ha) + $40 \%$ RDN through chemical fertilizer (urea $535.73 \mathrm{~kg} / \mathrm{ha}$ ) + GM (sunnhemp@ 8.88 t/ha) and Azospirillum (@ $30.86 \mathrm{~kg} / \mathrm{ha}$ ) equivalent to $20 \%$ RDN + PSB (@ $30.86 \mathrm{~kg} / \mathrm{ha})$.

$\mathrm{T}_{8 \text {. }}$ PM equivalent to $40 \%$ RDN (8.96 t/ha)+ $40 \%$ RDN through chemical fertilizer (urea $535.73 \mathrm{~kg} / \mathrm{ha}$ ) + GM (sunnhemp @ 8.88 t/ha) and Azospirillum (@ $30.86 \mathrm{~kg} / \mathrm{ha}$ ) equivalent to $20 \%$ RDN + PSB (@30.86 kg/ha).

T. SM equivalent to $40 \%$ RDN (10.17 t/ha) + $40 \%$ RDN through chemical fertilizer (urea $535.73 \mathrm{~kg} / \mathrm{ha}$ ) + GM (sunnhemp @ $8.88 \mathrm{t} / \mathrm{ha})$ and Azospirillum (@ $30.86 \mathrm{~kg} / \mathrm{ha}$ ) equivalent to $20 \% \mathrm{RDN}+$ PSB (@30.86 kg/ha).

$\mathrm{T}_{10}$ - AG equivalent to $40 \% \mathrm{RDN}$ (10.52 t/ha) + 40 \% RDN through chemical fertilizer (urea $535.73 \mathrm{~kg} / \mathrm{ha}$ ) + GM (sunnhemp @ 8.88 t/ha) and Azospirillum (@ $30.86 \mathrm{~kg} / \mathrm{ha}$ ) equivalent to 20\% RDN + PSB (@30.86 kg/ha

$\mathrm{T}_{11}$. BL equivalent to $40 \% \mathrm{RDN}$ (7.56 t/ha) + $40 \%$ RDN through chemical fertilizer (urea $535.73 \mathrm{~kg} / \mathrm{ha}$ ) + GM (sunnhemp @ 8.88 t/ha) and Azospirillum (@ $30.86 \mathrm{~kg} / \mathrm{ha}$ ) equivalent to $20 \% \mathrm{RDN}+$ PSB (@ $30.86 \mathrm{~kg} / \mathrm{ha})$

$\mathrm{T}_{12}$-Control (RDF=617.20:308.60: $925.80 \mathrm{~kg} \mathrm{NPK} / \mathrm{ha}+$ Farm yard manure @ $40 \mathrm{t} / \mathrm{ha}$ (plant crop)

Ratoon crop: RDF=308.60:154.20: 308.60 kg NPK/ha) + FYM @ 20/ha accordingly it has been supplied through different sources

FYM- Farmyard manure, VC-Vermicompost, PM-Poultry manure, SM-Sheep manure, AG-Agrigold, BL-Bhumilabh, GM-Green manure, PSB-Phosphate Solubilizing bacteria 
Table.4 Influence of different sources of nutrient modules on quality parameters in banana cv. Grand Naine (Pooled mean)

\begin{tabular}{|c|c|c|c|c|c|c|c|c|}
\hline Treatments & $\begin{array}{c}\text { TSS } \\
\left({ }^{0} \text { brix }\right)\end{array}$ & $\begin{array}{c}\text { Total Sugars } \\
(\%)\end{array}$ & $\begin{array}{c}\text { RS } \\
(\%)\end{array}$ & $\begin{array}{l}\text { NRS } \\
(\%)\end{array}$ & $\begin{array}{c}\text { Starch } \\
(\%)\end{array}$ & $\begin{array}{c}\text { Titrable acidity } \\
(\%)\end{array}$ & $\begin{array}{l}\text { Pulp to } \\
\text { peel ratio }\end{array}$ & $\begin{array}{l}\text { Shelf life } \\
\text { (Days) }\end{array}$ \\
\hline$T_{1}$ & 19.82 & 18.95 & 16.92 & 2.03 & 5.00 & 0.33 & 3.25 & 5.04 \\
\hline $\mathbf{T}_{2}$ & 19.46 & 18.25 & 16.55 & 1.70 & 5.74 & 0.36 & 2.78 & 4.55 \\
\hline $\mathbf{T}_{3}$ & 19.02 & 17.88 & 16.31 & 1.57 & 6.08 & 0.37 & 2.55 & 4.53 \\
\hline $\mathbf{T}_{4}$ & 19.54 & 18.57 & 16.80 & 1.77 & 5.00 & 0.33 & 3.12 & 5.04 \\
\hline $\mathbf{T}_{5}$ & 19.70 & 18.76 & 16.97 & 1.80 & 5.17 & 0.33 & 3.04 & 4.76 \\
\hline $\mathbf{T}_{6}$ & 21.45 & 19.49 & 17.30 & 2.19 & 4.33 & 0.29 & 3.47 & 5.41 \\
\hline $\mathbf{T}_{7}$ & 23.52 & 20.30 & 17.87 & 2.43 & 3.67 & 0.25 & 3.81 & 6.33 \\
\hline $\mathrm{T}_{8}$ & 19.13 & 17.52 & 15.98 & 1.54 & 6.21 & 0.39 & 2.46 & 4.12 \\
\hline $\mathbf{T}_{9}$ & 20.25 & 19.07 & 17.03 & 2.04 & 4.92 & 0.32 & 3.33 & 5.38 \\
\hline$T_{10}$ & 22.23 & 20.10 & 17.71 & 2.39 & 3.67 & 0.25 & 3.74 & 5.80 \\
\hline $\mathbf{T}_{11}$ & 22.07 & 19.31 & 17.09 & 2.22 & 3.84 & 0.28 & 3.56 & 5.68 \\
\hline $\mathbf{T}_{12}$ & 20.57 & 19.13 & 16.98 & 2.15 & 4.48 & 0.32 & 3.35 & 5.46 \\
\hline S Em+/- & 0.20 & 0.39 & 0.39 & 0.02 & 0.05 & 0.00 & 0.04 & 0.08 \\
\hline CD at $5 \%$ & 0.58 & 1.15 & 1.13 & 0.06 & 0.16 & 0.01 & 0.10 & 0.24 \\
\hline
\end{tabular}

T 1 FYM equivalent to $40 \%$ RDN (48.40 t/ha) + VC equivalent to $40 \%$ RDN (24.20 t/ha)+GM (sunnhemp @ 8.88 t/ha) and Azospirillum (@ 30.86 kg/ha) equivalent to $20 \%$ RDN + PSB (@ 30.86 $\mathrm{kg} / \mathrm{ha}$.

$\mathrm{T}_{2}$-FYM equivalent to $40 \%$ RDN (48.40 t/ha) + PM equivalent to $40 \%$ RDN (8.96 t/ha)+ GM (sunnhemp @ 8.88 t/ha) and Azospirillum (@ 30.86 kg/ha) equivalent to $20 \%$ RDN + PSB (@ 30.86 $\mathrm{kg} / \mathrm{ha})$.

T3- FYM equivalent to 40 \% RDN (48.40 t/ha) + SM equivalent to 40 \% RDN (10.17 t/ha) + GM (sunnhemp @ 8.88 t/ha) and Azospirillum (@30.86 kg/ha) equivalent to 20 \% RDN + PSB (@ 30.86

T. FYM equivalent to 40 \% RDN (48.40 t/ha) + AG equivalent to 40 \% RDN (10.52 t/ha) + GM (sunnhemp @ 8.88 t/ha) and Azospirillum (@ 30.86 kg/ha) equivalent to $20 \%$ RDN + PSB (@ 30.86 $\mathrm{T}_{4} \mathrm{FY}$

$\mathrm{kg} / \mathrm{ha}$ ).

T5- FYM equivalent to 40 \% RDN (48.40 t/ha) + BL equivalent to 40 \% RDN (7.56 t/ha)+ GM (Sunnhemp @ 8.88 t/ha) and Azospirillum (@ 30.86 kg/ha) equivalent to 20 \% RDN + PSB (@30.86 $\mathrm{kg} / \mathrm{ha})$.

$\mathrm{T}_{6}$ FYM equivalent to $40 \%$ RDN (48.40 t/ha) + 40 \% RDN through chemical fertilizer (urea $535.73 \mathrm{~kg} / \mathrm{ha}$ ) + GM (sunnhemp @ 8.88 t/ha) and Azospirillum (@ $30.86 \mathrm{~kg} / \mathrm{ha}$ ) equivalent to 20\% RDN + PSB (@ $30.86 \mathrm{~kg} / \mathrm{ha})$.

T 7. VC equivalent to $40 \%$ RDN (24.20 t/ha) + 40 \% RDN through chemical fertilizer (urea $535.73 \mathrm{~kg} / \mathrm{ha}$ ) + GM (sunnhemp@ 8.88 t/ha) and Azospirillum (@ $30.86 \mathrm{~kg} / \mathrm{ha}$ ) equivalent to $20 \%$ RDN + PSB (@30.86 kg/ha).

$\mathrm{T}_{8 \text { - }}$ PM equivalent to $40 \% \mathrm{RDN}$ (8.96 t/ha)+ 40 \% RDN through chemical fertilizer (urea $535.73 \mathrm{~kg} / \mathrm{ha}$ ) + GM (sunnhemp @ 8.88 t/ha) and Azospirillum (@ $\left.30.86 \mathrm{~kg} / \mathrm{ha}\right)$ equivalent to $20 \% \mathrm{RDN}+$ PSB (@ $30.86 \mathrm{~kg} / \mathrm{ha})$.

$\mathrm{T}_{\text {9. }}$ SM equivalent to $40 \% \mathrm{RDN}$ (10.17 t/ha) + $40 \%$ RDN through chemical fertilizer (urea $535.73 \mathrm{~kg} / \mathrm{ha}$ ) + GM (sunnhemp @ 8.88 t/ha) and Azospirillum (@ $30.86 \mathrm{~kg} / \mathrm{ha}$ ) equivalent to $20 \%$ RDN + PSB (@ $30.86 \mathrm{~kg} / \mathrm{ha})$.

$\mathrm{T}_{10}$ - AG equivalent to $40 \% \mathrm{RDN}(10.52 \mathrm{t} / \mathrm{ha})+40 \% \mathrm{RDN}$ through chemical fertilizer (urea $\left.535.73 \mathrm{~kg} / \mathrm{ha}\right)+\mathrm{GM}$ (sunnhemp @ 8.88 t/ha) and Azospirillum (@ $\left.30.86 \mathrm{~kg} / \mathrm{ha}\right)$ equivalent to $20 \% \mathrm{RDN}+$ PSB (@30.86 kg/ha

$\mathrm{T}_{11}$. BL equivalent to $40 \%$ RDN (7.56 t/ha) + $40 \%$ RDN through chemical fertilizer (urea $535.73 \mathrm{~kg} / \mathrm{ha}$ ) + GM (sunnhemp @ 8.88 t/ha) and Azospirillum (@ $30.86 \mathrm{~kg} / \mathrm{ha}$ ) equivalent to $20 \%$ RDN + PSB (@30.86 kg/ha)

$\mathrm{T}_{12}$-Control (RDF=617.20:308.60: $925.80 \mathrm{~kg} \mathrm{NPK} / \mathrm{ha}+$ Farm yard manure@ $40 \mathrm{t} / \mathrm{ha}$ (plant crop)

Ratoon crop: RDF=308.60:154.20: 308.60 kg NPK/ha) + FYM @ 20/ha accordingly it has been supplied through different sources

FYM- Farmyard manure, VC-Vermicompost, PM-Poultry manure, SM-Sheep manure, AG-Agrigold, BL-Bhumilabh, GM-Green manure, PSB-Phosphate Solubilizing bacteria 
Hazarika et al., (2011) reported that, the highest number of fingers per hand (22.87), finger length $(22.97 \mathrm{~cm})$, circumference $(14.13 \mathrm{~cm})$, finger volume $(282.98 \mathrm{cc})$ and weight of finger $(231.67 \mathrm{~g})$ were significantly influenced by $100 \%$ RDF +VAM (50 g/plant) + Azospirillum (50 g/plant) + PSB (50 $\mathrm{g} /$ plant $)+$ Trichoderma harjianum (50 $\mathrm{g} /$ plant) in banana.

Patil and Shinde (2013) recorded the highest bunch weight of $19.31 \mathrm{~kg}$ in plants inoculated with $75 \%$ RDF + FYM + Azotobacter $(50 \mathrm{~g}$ /plant) + PSB (50 g /plant) + VAM (250 $\mathrm{g} /$ plant) in Ardhapuri cultivar of banana. They also reported that, the highest yield of 85.80 t/ha obtained when the plants were treated with $50 \% \mathrm{RDF}+\mathrm{FYM}+$ Azatobacter (50 g/plant) + PSB (50 g/plant) + VAM (250 g/plant) in banana cv. Ardhapuri (AAA). Significant increase in number of fingers per bunch and average bunch weight of $5.0 \mathrm{~kg}$ by applying vermicompost $+\mathrm{N}$ as inorganic fertilizer in banana reported by Manivannan and Selvamani (2014). They also reported that, maximum bunch weight, more number of fingers per bunch was obtained by applying vermicompost as full $\mathrm{N}$ in banana. They also opined that, significantly more finger weight and finger girth was obtained by applying organic + inorganic fertilizers. The maximum number of fingers per bunch, bunch weight $(5.10 \mathrm{~kg} / \mathrm{bunch})$ and finally fruit yield $(15.14$ t/ha) was obtained by applying $50 \% \mathrm{RDF}+$ vermicompost $2.00 \mathrm{~kg} /$ plant in banana.

\section{Influence of different sources of nutrient modules on quality parameters (Table 4)}

The mean of the pooled data indicated that, the quality parameters like Total Soluble Solids, Reducing sugars, Non reducing sugars, Total Sugars, Pulp to peel ratio and Shelf life was maximum $\left(23.52^{0} \mathrm{~B}, 17.87 \%\right.$, $2.43 \%, 20.31 \%, 3.81$ and 6.33 days respectively) in banana fruits harvested from plants treated with $\mathrm{T}_{7}$ \{Vermicompost equivalent to $40 \% \operatorname{RDN}(24.20 \mathrm{t} / \mathrm{ha})+40 \%$ RDN through chemical fertilizer (urea 535.73 kg/ ha) + Green manure (sunnhemp @ 8.88 t/ha) and Azospirillum (30.86 kg/ha) equivalent to $20 \% \mathrm{RDN}+\mathrm{PSB}$ (30.86 $\mathrm{kg} / \mathrm{ha})$. Whereas, the least titratable acidity $(0.25 \%)$ was recorded in $\mathrm{T}_{7}$ and $\mathrm{T}_{10}$ treatments followed by $\mathrm{T}_{11}$ treatment. However, the starch was least $(3.67 \%)$ in $\mathrm{T}_{7}$ and $\mathrm{T}_{10}$ treatments. In general the fruit quality parameters were improved in integrated nutrient modules compared to organic treatments.

The main changes in fruit pulp during ripening are the conversion of starch to sugars. As ripening proceeds, pulp to peel ratio was increased from 2.0 in stage 5 to 2.7 in stage 7 when the fruits become fully ripened.

This could be due to the osmotic transfer of moisture from the peel to the pulp as sugar content of pulp increased. It has been suggested that pulp to peel ratio can be considered as a coefficient of ripeness. These changes during ripening period (loss of greenness and increase in yellowness) may occur as a result of the breakdown of the chlorophyll in the peel tissue.

Venkatesh (1995) observed that application of organic manures viz., vermicompost (@4 t/ha) and farmyard manure (@ 2.5 t/ha) resulted in significantly higher amount of total sugars and decreased titratable acidity compared to inorganic fertilizers in grape. The results of present study also indicated the same. The results indicated that a progressive increase in total sugar content and decrease in starch content was observed during ripening. Thompson (1996) reported that the softening of banana fruit during ripening is associated with the conversion of starch to sugar, breakdown of pectin substances and the 
movement of water from rind of banana to the pulp and this could be the major cause for decreasing the various mechanical properties of banana during ripening. Ushakumari et al., (1997) recorded similar results as that of present study. They obtained significantly more reducing sugar by applying vermicompost as full $\mathrm{N}$ in banana cv. Njalipoovan.

They also reported that significantly higher content of total sugar was obtained by applying vermicompost + inorganic fertilizers whereas, significantly less acidity was obtained by applying vermicompost + inorganic fertilizers and vermiculture in-situ and the highest content of non-reducing sugar by applying vermiculture in-situ. This could be due to the hydrolysis of starch into sugar during ripening (Garcia and Lajolo, 1988). Increase in TSS is an important trait of hydrolysis of starch in to soluble sugars such as glucose, sucrose and fructose (Stover and Simmonds, 1987 and Kulkarni et al., 2010).

Mustaffa et al., (2004) opined that application of $2.5 \mathrm{~kg}$ compost $+1.0 \mathrm{~kg}$ vermicompost + $1.0 \mathrm{~kg}$ neem cake $+2.5 \mathrm{~kg}$ poultry manure at 3, 5 and 7 months after planting recorded the maximum TSS $\left(29.40^{\circ}\right.$ Brix and $32.20^{\circ}$ Brix respectively), acidity $(0.59 \%$ and $0.61 \%$ respectively), sugar - acid ratio (49.8 and 52.8 respectively), total sugars $(25 \%$ and $26.3 \%$ respectively) and low starch $(3.2 \%$ and $3.4 \%$ respectively). The lowest quality was recorded in inorganically fertilized fruits of Rasthali and Karpuravalli cultivars of banana respectively.

Hazarika and Ansari (2008) revealed that biochemical constituents viz., TSS, reducing sugar, non-reducing sugar, ascorbic acid, and moisture content of fruits was significantly influenced by different treatments having organic manure and bio-fertilizer along with inorganic fertilizers except sugar-acid ratio and titratable acidity in banana cv. Jahaji (AAA).

The results of Tangaselvabai et al., (2009) were in line with the present study. They reported that the highest TSS, reducing sugars, non-reducing sugars, total sugars, ascorbic acid and starch was noticed in plants treated with 100:30:330 g NPK/plant in 2 splits + Azospirillum, 100:30:330 g $\mathrm{NPK} /$ plant in 2 splits + Azospirillum, 75:30:330 g NPK/plant in 2 splits + Azospirillum, 220:30:330 g NPK/plant in 4 splits, 100:30:330 g NPK/plant in 2 splits + Azospirillum and 100:30:330 g NPK/plant in 2 splits+ Azospirillum respectively.

Application of plants with vermicompost equivalent to $40 \% \mathrm{RDN}(24.20 \mathrm{t} / \mathrm{ha})+40 \%$ RDN through chemical fertilizer (urea 535.73 $\mathrm{kg} / \mathrm{ha}$ ) + Green manure (sunnhemp @ 8.88 t/ha) and Azospirillum (30.86 kg/ha) equivalent to $20 \% \mathrm{RDN}+\mathrm{PSB}(30.86 \mathrm{~kg} / \mathrm{ha})$ $\left(\mathrm{T}_{7}\right)$ was most superior in increasing the growth, yield and quality parameters.

The integrated nutrient modules favoured higher growth, yield and quality parameters than organic modules followed by $\mathrm{T}_{10}$ (with Agri-gold combination) and $\mathrm{T}_{11}$ (with Bhumilabh combination) indicating the potentiality of these organic manures when used in conjunction with chemical and biofertilizers.

\section{References}

Anonymous, 2011. Indian Horticulture data base, National Horticulture Board. Department of Agriculture and Cooperation, Ministry of Agriculture, Govt. of India, New Delhi. pp 34-41

Athani S I, Hulamanai N C and Shirol A M 1999. Effect of vermicompost on maturity and yield of banana cv. Rajapuri (Musa AAB). South Indian Hort. 47(1-6): 4-7.

Garcia, E. and Lajolo, F. M., 1988. Starch 
transformation during banana ripening: the amylase and glucosidase behavior. J. Food Sci., 53: 1181-1188.

Hazarika, B. N. and Ansari, S., 2008. Effect of integrated nutrient management on quality of banana cv. Jahaji (AAA). Indian Agriculturist, 52(3/4): 175-178.

Hazarika, T. K., Bhattacharyya, R. K. and Nautiyal, B. P., 2015. Growth Parameters, Leaf characteristics and Nutrient Status of Banana as Influenced by Organics, Bio-fertilizers and Bio-agents. Journal of Plant Nutrition, 38 (8).

Hazarika, T. K., Nautiyal, B. P. and Bhattacharya, R. K., 2011. Effect of INM on productivity and soil characteristics of tissue cultured banana cv. Grand Naine in Mizoram, India. Indian J. Hort. 43(1): 30-35.

Jayabaskaran, K. J., Pandey, S. D., Mustaffa, M. M. and Sathiamoorthy, S., 2001. Effect of different organic manures with graded levels of inorganic fertilizers on ratoon of Poovan banana. South Indian Hort. 49: 105-108.

Kulkarni, S. G., Kudachikar, V.B. and Prakash, M. N., 2010. Studies on physico- chemical changes during artificial ripening of banana (Musa sp) variety 'Robusta'. J Food Sci Technol. (online journal DOI 10.1007/s13197-010-0133-y)

Manivannan, K. and Selvamani, P., 2014. Influence of organic inputs on the yield and quality of fruits in banana cultivar 'Poovan' (Syn. Mysore AAB). ISHS Acta Hortic. 1018.

Mustaffa, M. M., Kumar, V., Tanuja Priya, B. and Dhanasekhar, D., 2004. Influence of organic manure on growth and yield of banana. International congress on Musa:
Harnessing research to improve livelihoods, Penang, Malaysia. 214: 65-66.

Nachegowda V, Senthil Kumar $K$ and Raghavendra Prasad G C 2004. Effect of organic and inorganic fertilizers on growth, yield and quality of tissue cultured banana cv. Robusta. Proceedings of National Seminar on Banana Industry - Present Scenario and Future Strategies held at BCKV, FTC, Kalyani, West Bengal, 11 $13^{\text {th }}$ June, 2004.

Patil, V. K. and Shinde, B. N., 2013. Studies on integrated nutrient management on growth and yield of banana cv. Ardhapuri (Musa AAA). J. Hort. For., 5(9):130-138.

Stover, R. H. and Simmonds, N. W., 1987. Bananas. $3^{\text {rd }}$ Ed.; John, W. \& Sons Inc: New York. p. 256

Tangaselvabai, T., Gailice leo justin, C., Nirmal Johnson, S.B. and Jayasekhar, M., 2009. Influence of nutrients on qualitative and quantitative traits of banana. Indian $J$. Agric. Res. 43(4): 274-278.

Thompson, A. K., 1996. Postharvest treatments. In: Postharvest Technology of Fruit and Vegetables, Blackwell Publishing. Oxford, pp. $95-124$

Ushakumari, K., Prabhukumari, P. and Padmaja, P. 1997. Efficiency of vermicompost on yield and quality of banana (AB) cv. Njalipoovan. South Indian Hort., 49(34):158-160.

Venkatesh, 1995. Effect of vermiculture on soil composition, growth, yield and quality of Thomson seedless grapes (Vitis vinifera L.). M.Sc. (Agri.) Thesis University of Agricultural Sciences, Dharwad.

\section{How to cite this article:}

Ganapathi, T. and Dharmatti, P.R. 2018. Effect of Integrated Nutrient Modules on Growth, Yield and Quality Parameters of Banana cv. Grand Naine. Int.J.Curr.Microbiol.App.Sci. 7(01): 1974-1984. doi: https://doi.org/10.20546/ijcmas.2018.701.239 